\title{
Eye Localization Based on Maximal Complexity of Rotating Scan Lines
}

\author{
Sanliang Hong ${ }^{\mathrm{a}}$,Qingxiang $\mathrm{Wu}^{\mathrm{b}}{ }^{*}$,Binshu $\mathrm{Chen}^{\mathrm{c}}$,Liuping \\ Huang $^{\mathrm{d}}$,Yanfeng Chen ${ }^{\mathrm{e}}$ \\ Key Laboratory of OptoElectronic Science and Technology for Medicine \\ of Ministry of Education, College of Photonic and Electronic Engineeri \\ ng, Fujian Normal University, Fuzhou, China ${ }^{a} 913452977 @ q q . c o m,{ }^{b} q x$ \\ wu@fjnu.edu.cn, ${ }^{c} h a a r \_l i k e @ o u t l o o k . c o m,{ }^{d} h l p 590045 @ 163 . c o m,{ }^{e} 474741$ \\ 702@qq.com
}

\begin{abstract}
A novel algorithm based on maximal grayscale complexity of rotating scan lines is proposed to locate the human eye in the detected human face region. First of all, the human face regions are detected from a number of outdoor photos using the skin segmentation algorithm. Secondly, the skin block is retained in the human face region employing the skin block extraction algorithm. Finally, the grayscale complexity is computed by rotating scan line in these skin blocks and the human eyes are located at the straight line corresponding to the maximum of grayscale complexity. The proposed algorithm has a good robustness of the variation of low-resolution faces with head postures and accessories and it is much better than other approaches.
\end{abstract}

Keywords: skin segmentation, eye localization, scan line rotation, maximal grayscale complexity

\section{Introduction}

Eye localization plays a significant role in face recognition, intelligent monitoring, video tracking and the like. However, a set of factors bring great difficulty to eye localization such as low-resolution face (the pupil and the whites of the eyes cannot be distinguished), head tilt, the face occlusion of masks, scarves, sunglasses, hats and other accessories or hands and the strong, weak or uneven illumination, etc. So far, many eye localization methods have been put forward. The method based on grayscale integration projection used in [1]. Face images are horizontal and vertical integrated and eye localization is based on the projection curve distribution, however, the eye localization is easily affected by head posture and the eye positioning is not accurate because of the complicated information distribution of image grayscale. The Gabor model employed in [2] is able to overcome noise effectively, but the eyes are difficult to be located when there is hair, glasses and other accessories interference. The method of Hough transform is used in $[3,4]$, wherein firstly locates the eye area in binary image 
roughly, then the edge analysis is performed in the region, finally the pupil center position is located by Hough transform. The localization accuracy is largely influenced by the eyeball with this method and it is inapplicable to low-resolution face. Additionally, the method based on the combination of the gray projection and the LMS template matching used in[5], the approach based on adaptive image segmentation and curve blending employed in [6] are also utilized to eye localization, but some results of these methods are unsatisfactory for low resolution faces.

In order to overcome the influence of the eyes' being not on the horizontal line caused by head tilt, or low-resolution face, eye glasses and other accessories in eye localization, this paper presents an eye localization algorithm based on maximum of grayscale complexity with rotation of scan line. Because the position of the eyes is basically in the upper part of the face area and the complexity of the eyes is relatively large, the scan algorithm is only used in the upper half of the face image. First of all, all the human face areas are detected from a number of outdoor photos using the skin segmentation algorithm. Secondly, only the skin block in the human face area is retained by employing the skin block extraction algorithm. Finally, the grayscale complexity is computed by rotating scan line in these skin blocks and the human eyes are located at the straight line corresponding to the maximal grayscale complexity.

\section{Face region detection based on YCbCr color model algorithm}

Color space

Face detection usually uses RGB, YCbCr, YIQ and HSV color space. In this paper, the skin segmentation is based on YCbCr color space. In YCbCr space, Y represents luminance information of the color component. $\mathrm{Cr}$ and $\mathrm{Cb}$ represent red and blue components of the color information respectively. The formula (1) expresses conversion from RGB space to YCbCr space in [7]

$$
\left[\begin{array}{c}
Y \\
C b \\
C r
\end{array}\right]=\left[\begin{array}{c}
16 \\
128 \\
128
\end{array}\right]+\left[\begin{array}{ccc}
65.481 & 128.553 & 24.966 \\
-37.797 & -74.203 & 112.000 \\
112.000 & -93.786 & -18.214
\end{array}\right]\left[\begin{array}{l}
R \\
G \\
B
\end{array}\right]
$$

Skin segmentation

In the YCbCr space, the skin information $\mathrm{Cb}$ and $\mathrm{Cr}$ are within a relatively stable distribution of $90<\mathrm{Cb}<127,135<\mathrm{Cr}<177$, cited in [8], The region which satisfies the range of skin in YCbCr space is set to white, and the others are set to black. Then a binary image after skin segmentation is obtained in this way. 


\section{Adjustable structural elements preprocessing}

Pretreatment is a significant part during face detection process and it directly affects the ultimate face detection rate. Since using the fixed structural elements to preprocess binary image will lose some faces, adjustable structural elements preprocessing [9] is used to reduce the lost faces. As face shape is similar to oval, adjustable disc structural elements preprocessing is used in this paper. Selecting adjustable structural elements can be described as following steps:

1)Mark all connected regions in the binary image that has been skin segmented;

2)Calculate the area of all the connected regions $S$;

3)Calculate the average of all areas of connected regions $s^{\prime}$;

4)The radius of the disk-shaped structure element $r=a^{*} s^{\prime}$, herein $r$ varies with variable $a$.

The connected regions are removed if the radius is less than $r$. As $a$ is increasing, the $r$ is incremented, the number of connected regions decline sharply, especially the little regions regarded as noises are removed. The number of connected region reaches a stable number when variable $a$ reaches at $a=0.009$. According to the respective four boundaries of the retained connected regions, the blocks are cut out from the image corresponding to face regions.

The result is shown in Figure 1. Figure 1(a) is an original image, Figure 1(b) is the pretreatment result. Figure 1(c) is the detected face blocks.

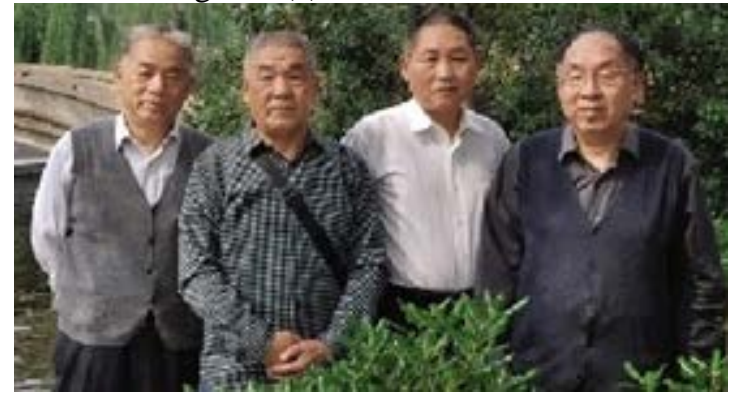

(a) original image

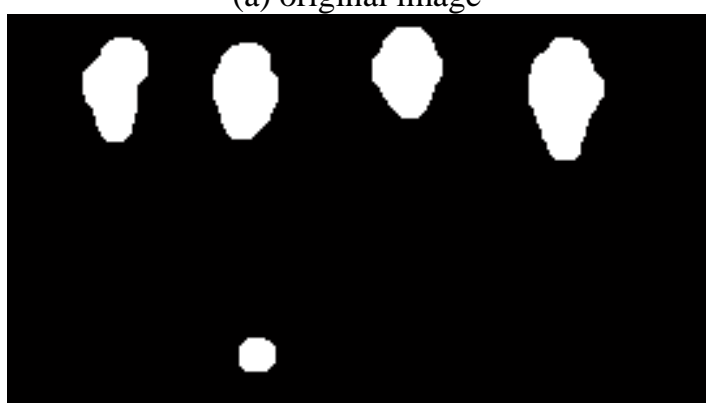

(b) pretreatment result 

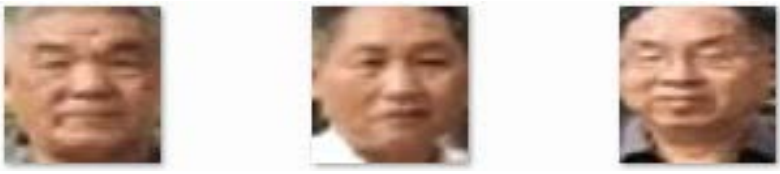

(c) detected face blocks

Figure 1 Results of skin segmentation and face block extraction

\section{Eye localization based on grayscale complexity with scan line rotation}

The following formula (2) [10] is used to calculate grayscale complexity.

$$
\lambda=\sum_{i=1}^{m} \sum_{j=2}^{n}|R(i, j)-R(i, j-1)|
$$

According to the detected face regions, because of head tilt, there is an intersecting angle between binocular connection line and horizontal line. It could be chosen to rotate the skin block so that binocular can be accordance with a horizontal line. However, after rotation the grayscale of skin will redistribute. Consequently, scan line rotation algorithm is presented to improve this situation in this paper. The so-called scan line rotation is that the scan line is rotated with a slope. Since the human eye is generally on the upper part of the face region, so just the upper half of the face images is selected as part of the region of interest $(R O I)$. Common eye positions in ROI are shown in Figure 2(a), Figure 2(b) and Figure 2(c):

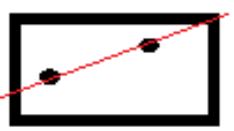

(a)

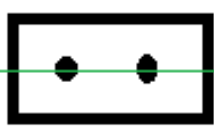

(b)

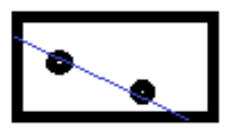

(c)

Figure 2 Three situations of head tilt

As shown in Figure 2(a), binocular connection line intersects with the top and the left edge of $R O I$, the scan line is determined by the top and the left edge of ROI. The size of ROI is $m^{*} n$. Herein the scan line is determined by formula (3).

$$
y_{1}=k_{1 i}^{j} *(p-1)+i
$$

where $k_{1 i}^{j}=\frac{1-i}{j-1}, i=4: m, j=\frac{2 n}{3}: n, p=2: j$.

When binocular connection line intersects with the right and the left edge of ROI, as shown in Figure 2(b), the scan line is determined by the right and the left 
edge of ROI. The scan line is determined by formula (4).

$$
y_{2}=k_{2 i}{ }^{t}(j-1)+i
$$

where $k_{2 i}{ }^{t}=\frac{t-i}{n-1}, i=1: m, t=2: m-1, j=2: n$.

When binocular connection line intersects with the bottom and the left edge of ROI, the line, as shown in Figure 2(c), represents the scan line that is determined by the bottom and the left edge of $R O I$. The scan line is determined by formula (5).

$$
y_{3}=k_{3 i}^{j} *(p-1)+i
$$

where $k_{3 i}^{j}=\frac{m-i}{j-1}, i=1: m, j=\frac{2 n}{3}: n, p=2: j$.

The extreme cases that serious head tilting to the right makes binocular connection intersect with the top and the right edge of $R O I$ or with the bottom and the right edge of $R O I$ rarely occur. Therefore, these cases are not considered.

According to formula (3), formula (4) and formula (5), the scan line is rotated. When the scan line coincides with binocular connection line or has a very small intersecting angle with binocular connection line, the maximal grayscale complexity can be obtained along the scan line. Thus the eye localization is determined.

The concrete description of algorithm as follows: in Figure 2(a), scan line is determined by $y_{1}$, every $y_{1}$ denotes a straight line, then the corresponding grayscale complexity is computed along the straight line. The corresponding calculation formula is shown as formula (6):

$$
\left.\lambda_{k_{1 i}^{j}}=\sum_{i=3}^{m} \sum_{p=2}^{j} \mid R\left(k_{1 i}^{j} *(p-1)+i\right), p\right)-R\left(k_{1 i}^{j} *(p-2)+i, p-1\right) \mid\left(j=\frac{2 n}{3}: n\right)
$$

And the like, corresponding straight lines are determined by $y_{2}$ and $y_{3}$ in Figure 2(b) and in Figure 2(c) respectively. The corresponding calculation formula is shown as formula (7) and formula (8) respectively.

$$
\begin{aligned}
& \left.\lambda_{k_{2 i}^{j}}=\sum_{i=1}^{m} \sum_{j=2}^{n} \mid R\left(k_{2 i}{ }^{t} *(j-1)+i\right), j\right)-R\left(k_{2 i}{ }^{t} *(j-2)+i, j-1\right) \mid(t=2,3 \ldots m-1) \\
& \left.\lambda_{k_{3 i}^{j}}=\sum_{i=1}^{m} \sum_{p=2}^{j} \mid R\left(k_{3 i}^{j} *(p-1)+i\right), p\right)-R\left(k_{3 i}^{j} *(p-2)+i, p-1\right) \mid\left(j=\frac{2 n}{3}: n\right)
\end{aligned}
$$

Grayscale complexity calculation is carried on along these straight lines 
respectively. Then the maximal grayscale complexity is found out and the straight line with maximal complicity is determined as eye position. Draw this line and locate the human eye.

\section{The experimental results and analysis}

For the purpose of verifying the effectiveness of the algorithm, the human face region extraction is carried on in Matlab environment. The human face images for test contain different resolutions, head postures and accessories. Eye localization result is expressed by a straight line which goes through the eyes on skin block. It's shown in Figure 3.

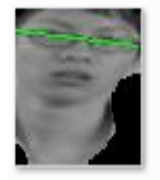

foo1

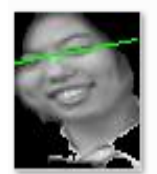

fo06

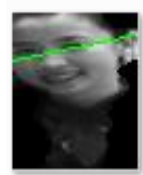

fo11

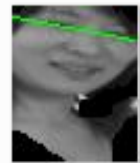

fo02

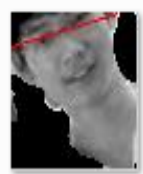

f_007

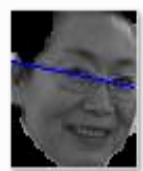

f_012

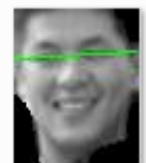

f_003

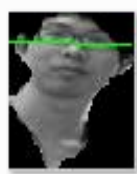

f_o08

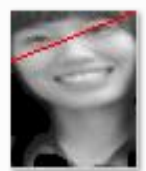

fo13

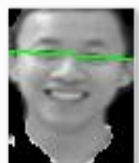

f_004

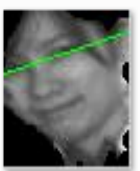

f_o09

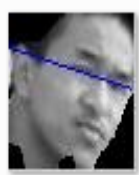

f.014

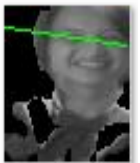

f_o05

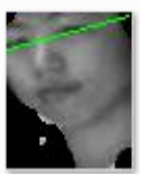

f_010

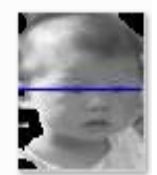

fo15

Figure 3 Eye localization

As can be seen by Figure 3, the proposed algorithm for eye localization has better applicability; it is capable of locating the eyes basically. Positioning accuracy rate can reach $91 \%$. The algorithm overcomes the positioning difficulties caused by head tilt and the impact caused by eye brows. Also, it can achieve eye localization though serious head tilt exists and achieve eye localization in some low-resolution human face images.

Compared with the proposed algorithm, the method based on grayscale integration projection mentioned in the introduction part is applicable to high-resolution faces(the pupil and the whites of the eyes can be distinguished), the result of eye localization by using this method in low-resolution face is not accuracy. It's shown in Figure 4. 


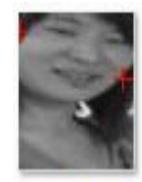

f-002

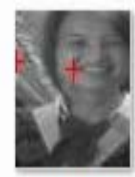

fo05

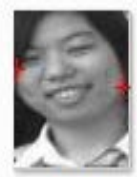

foo6

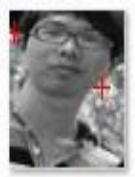

foos

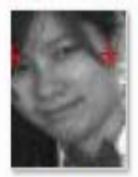

foog

Figure 4 Eye localization based on grayscale integration projection

However, the proposed algorithm also has some limitations. For instance, the strong or weak illumination causes skin segmentation defects and affects the positioning effect. When the background color is relatively close to skin, sometimes the localization effect can also be affected. The partial and very low resolution human faces are inclined to be hard to locate eyes, too.

\section{Conclusion}

In this paper, face region detection is based on YCbCr color model algorithm. During preprocessing, adjustable structural elements are utilized. Eye localization is based on grayscale complexity with scan line rotation. Experimental results show that the proposed algorithm for eye localization has better applicability and a relatively good robustness under the conditions that the head is tilted, the illumination is even, the background is relatively ideal and human face is integral. This algorithm can be applied in further face recognition research, as its limitations are the research issues to be solved in future.

\section{Acknowledgments.}

The authors gratefully acknowledge supports from Fujian Provincial Key Laboratory for Photonics Technology and the fund from the Natural Science Foundation of China (Grant No.61179011) and Science and Technology Major Projects for Industry-academic Cooperation of Universities in Fujian Province (Grant No.2013H6008).

\section{References}

[1] Karin Sobottka, Ioannis Pitas. A Novel Method for Automatic Face Segmentation, Facial Feature Extraction and Tracking. Signal Processing: Image Communication. Vol.12(1998), p.263-281

[2] Smeraldi F, O Carmona. Saccadic search with Gabor features applied to eye detection and real-time head tracking. Image and Vision Computing. Vol.18(2000), p.323-329.

[3] Canny J. A computational approach to edge detection. IEEE Transaction on Pattern Analysis and Machine Intelligence.Vol.8(1986), p.679-698.

[4] Zana'F. A multimodal registration algorithm of eye fundus images using vessels detection and Hough transform. IEEE Trans on Medical Imaging.Vol.18(1999), p.419-428. 
[5] Shu-bin Yang, Lu Jin, Zhen-bao Zhang. Fast eye location method in driver fatigue detection. J.Wuhan Inst.Tech. Vol.35(2013) No.6(In Chinese).

[6] Fan Yang, Hua Zhang, Guo-feng Pan,Zhi-tao Wang. Eye location based on adaptive image segmentation and curve blending. Optics and Precision Engineering.Vol.21(2013) No.12(In Chinese).

[7] Poynton, C. A.A Technical Introduction to Digital Video, John Wiley \& Sons, Inc., 1996, p. 175.

[8] Hong You, Ji-Zhu Nan. Using normal form of matrices over finite fields to construct Cartesian authentication codes. Journal of Mathematical Research \& Exposition.Vol.18(1998), p.341 - 346 (In Chinese).

[9] Wei-qi Yuan, Chun-xia Han. Multi-face detection algorithm in complex background. Journal of Computer App localizations.Vol.30(2010), p.635 640 (In Chinese).

[10] Lian-yan Cui, Lin Xu, Shu-sheng Gu. Eyes localization Based on the Calculation of the Complexity Extent and the Best Threshold Value. Control Engineering of China.Vol.15(2008), p.11-13 (In Chinese). 